\title{
Análise estatistica do ensaio mecânico de resistência à flexão em amostras de reciclagem de pneus
}

\section{Statistical analysis of the mechanical test of flexural strength in tire recycling samples}

\author{
André Hekermann Buss \\ Universidade Tecnológica Federal do Paraná - UTFPR - Ponta Grossa - Brasil \\ ahbuss@utfpr.edu.br \\ Regina Negri Pagani \\ Universidade Tecnológica Federal do Paraná - UTFPR - Ponta Grossa - Brasil \\ reginapagani@utfpr.edu.br \\ João Luiz Kovaleski \\ Universidade Tecnológica Federal do Paraná - UTFPR - Ponta Grossa - Brasil \\ kovaleski@utfpr.edu.br \\ Eriel Biagini Sabino \\ Universidade Tecnológica Federal do Paraná - UTFPR - Ponta Grossa - Brasil \\ eriel@utfpr.edu.br
}

\section{Resumo}

Este artigo mostra a importância do processo de reciclagem de pneus, registra os principais meios utilizados para este fim e resalva o método utilizado por uma patente junto ao Instituto de Propriedade Intelectual (INPI), ao mostrar os resultados do ensaio de resistência mecânica a flexão com $20 \%$ e $60 \%$ de adição, e carga junto a matriz polimérica de uma resina termofixa; a qual conforme literatura inclusive do Conselho Regional de Química (CR), indica que esta matriz polimérica não aceita quantidade muito superior a $30 \%$ de adição, portanto um método inovador. Os resultados dos ensaios de resistência à flexão foram mensurados por métodos estatísticos para comprovar qual quantidade de resíduos de pneus possuem os melhores resultados, e se está em conformidade com a literatura e estudos até o momento desenvolvidos.

Palavras-chave: Reciclagem de pneus, Pearson, Spearman.

\section{Abstract}

This article shows the importance of the tire recycling process, records the main means used for this purpose and highlights the method used by a patent with the Intellectual Property Institute (INPI), when showing the results of the flexural strength test with $20 \%$ and $60 \%$ addition, and charge together the polymer matrix of a thermoset resin; which according to literature including the Regional Council of Chemistry, indicates that this polymer matrix does not accept much more than 30\% addition, therefore an innovative method. The results of the flexural strength tests were measured by statistical methods to 
prove the amount of waste tires have the best results, and is in accordance with the literature and studies so far developed.

Keywords: Tire Recycling, Pearson, Spearman.

\section{Introdução}

Os pneus geram um problema ambiental quando chegam ao seu fim de vida, pois não existe atualmente um processo adequado ambientalmente que resulte numa solução para a reciclagem dos pneus (BERTOLLO; FERNANDES; SCHALCH, 2002). Já é comprovado que os pneus abandonados em córregos, na beira de estradas ou armazenados formando pilhas de pneus, além de gerarem risco a grandes incêndios, liberam o dióxido de carbono que podem contaminar lençóis de água contaminado o ambiente em torno (JON, 1994).

Os destinos mais comuns dos pneus usados e sem condições de uso no Brasil, são a sua queima em fornos; o que geram gases com as substâncias compostas junto à estrutura molecular que forma os pneus. Destaca-se que devem ser filtradas as partículas na sua totalidade para evitar danos ambienta (LAGARINHOS; TENÓRIO, 2009). Lagarinhos e Tenório (2009) já descrevem que os pneus inservíveis podem ser utilizados para alimentar os fornos de clínquer, onde reduzem entre 10\% e 30\% o uso de outros combustíveis nãorenováveis.

$\mathrm{Na}$ indústria de cimento, os pneus usados e sem uso, também podem alimentar os fornos promovendo o co-processamento, onde a malha de aço se incorpora ao clínquer, sendo um substituto da matéria prima, reduzindo os cstos operacionais (LAGARINHOS; TENÓRIO, 2009). Conforme Largarinhos e Tenório (2013), pode-se ter a informação de que em 2010 a indústria de pneumático fabricou aproximdamente 67,3 milhões de uundades de pneus, deste tem-se 23,9 milhões são importados e 18,1 milhões são exportados. O volume de pneus novos produzidos no Brasil, tem-se uma parcela que é utilizada para reposição nacional, sendo aproximadamente $45 \%$ do total, deste cerca de $30 \%$ é usado em veiculos novos e $25 \%$ para exportação (LAGARINHOS; TENÓRIO, 2013, p. 2).

De Brito (2004), cita a importância da logistica reversa como um sistema favorável de produção, que pode gerar aspectos tanto economicos como ambientais. Por sua vez, Freire e Guedes (2006) comentam sobre a eficacia e o quanto é eficiente a logística reversa, demonstrando a relevância deste processo para o meio ambiente ao auxiliar na reciclagem dos produtos, bem como sobre a visão da malhoria do nivel da produção industrial.

Existem leis que regulamentam o armazenamento e processo de fabricação de pneus nos Estados Unidos da América (EUA), onde há impedimento de pneus em aterros sanitários e por outro aspecto oferece incentivos para a utilização de novas tecnologias para reciclar os pneus em fim de vida (FHWA, 1993; EPA, 1999).

Desde 1999 o Brasil legislou conforme Resolução do CONAMA no. 258/99, que todo fabricante de pneus deveria dar destinação correta aos seus produtos, os quais são os pneus inservíveis; seguindo metas conforme sua produção. Esta Resolução ressalta que deve ser feita a coleta e dar destinação final, tendo abrangência aos produtores, distribuidores, revendedores, reformadores e incluindo os consumidores (LAGARINHOS; TENÓRIO, 2013).

Undri et al. (2013), mostra a dificuldade de se reciclar pneus devido as suas ligações químicas fortes e a grande composição de elementos envolvidos na reação química que possuem os pneus, sendo que esta composição é altamente tóxica e prejudicial à saúde humana e ao meio ambiente. 
Já há tempo se tenta dar um destino ambientalmente correto aos pneus inservíveis. Rodrigues (2008), cita que consegue aplicar resina de óleo de mamona adicionado em pedaços de borrachas de pneus inservíveis, sendo em seguido colocado sobre pressão em forma, produzindo placas antiderrapantes. Com isto, se tem um produto caracterizado por ser sustentável.

Dos diversos métodos utilizados para se reciclar borrachas de pneus inservíveis, tem-se os métodos conhecido por meio da criogenia, onde se usa de resfriamento rápido da borracha de pneus e seu trituramento logo em seguida. $O$ método por micro-ondas ocorre a quebra das cadeias químicas das borrachas de pneus, gerando pedaços menores promovendo a reciclagem desta borracha. No método por pirolise tem-se a evaporação dos pneus pela aplicação de pressão e vacuo em câmara especifica, onde se tem gás, o qual é condensado, e se têm como produto final diferentes óleos destinados para a indústria química. $O$ processo microbiológico ocorre quando há a utilização de microorganismos para decompor a borracha de pneus inservíveis.

O Conselho Regional de Química (CRQ, 2017), e o fabricante de resina termofixa (RECHHOLD, 2018), tem em seu sitio a citação de que resinas termofixas tem entre as principais características a resistência a radiação solar e aceitam carga na sua mistura em torno de $30 \%$ total da mistura.

Nota-se que as patentes são registros de estudos e fonte de demonstração das inovações tecnológicas, com isto é relevante se ter como referencia as patentes citadas como reciclagem de pneus, para se ter uma noção dos estudos mais recentes nessa área do conhecimento (INPI, 2017).

O principal objetivo deste estudo é revelar os resultados dos ensaios de flexão para amostras de resina termofixa com adição de $20 \%$ e $60 \%$ de resíduo de pneus, sendo estes analisados por métodos estatísticos para afirmar qual a melhor quantidade de adição de carga é mais vantajosa, com isto validar os resultados.

Para se atingir o objetivo geral é necessário ter noção da relevância da estatística e dos seus métodos, que são capazes de validar o processo em estudo (SARACEVIC, 2007).

Ramirez, Schau e Emmioğlu (2012), revelam a importância da estatística e o seu poder em validar os resultados de toda pesquisa, já que segue uma padronização equalizada para comparar os dados, originando em condições favoráveis e acertiva para afirmar as condições de análise dos resultados coletados.

Um dos fatores limitadores deste estudo é a falta de informações seguras e inovadoras, já que a área de pneus é muito competitiva e, portanto, há muito sigilo entre as empresas do setor e de quem investe em pesquisa.

Este trabalho esta estruturado com a introdução, citação das patentes sobre reciclagem de pneus, adição de carga de até $80 \%$ de concentração em resina termofixa, explanação dos métodos utilizados, ensaios mecânicos do protocolo de patente $\mathrm{N}^{\circ}$. BR10 2016 005015-4, resultados e discussões, conclusões.

\section{Patentes sobre reciclagem de pneus.}

Existem diferentes meios para se reciclar pneus, entre eles pode-se citar a criogenia, processo microbiológico, micro-onda ou eletromagnético e processo por pirolise (LENS, 2017; WIPO, 2017).

O processo de patente via criogenia, é um método que ocorre quando a borracha de pneus é envolvida por baixa temperatura muito abaixo de zero grau, e provoca a quebra da borracha de pneus ao sofrer impacto, por estarem duras devido ao resfriamento abrupto da borracha do pneu, resultando na formação de partículas bem pequenas. Neste processo, tem-se a patente US5634599, de 17 de fevereiro de 1994, intitulada Tire recycling process (WIPO, 2017). 
Já o processo que faz reciclagem de pneus por processo microbiológico citado na numeração US6. 479.558B1, de 2012, cujo nome oficial é Microbial processing of used rubber. Este processo descreve a utilização de micro-organismos chamados termófilos, que quebram as cadeias químicas e ramificadas das borrachas de pneus (LENS, 2017).

O processo via micro-ondas ou processo eletromagnético, é citado como exemplo a patente EP2993217 que gera desvulcanização, ou seja, a quebra das cadeias químicas da borracha, processo que tem o nome Devulcanization Of Rubber And Other Elastomers. Neste processo relata-se que os estudos foram concluídos em 12 de abril de 2017, e que trabalha com o processo de quebra de partículas de elastômero reticulado, que é a quebra da cadeia carbônica, pela utilização de um campo dielétrico de radio frequência usado com carga eletromagnética entre 1 e $100 \mathrm{MHz}$, e tensão entre 1000 e $10.000 \mathrm{~V}$, sendo direcionado diretamente nas borrachas de pneus (LENS, 2017).

O processo via pirolise ocorre quando se coloca pneus dentro de uma autoclave, e a máquina gera através de pressão e controle da temperatura a decomposição da borracha dos pneus, promovendo a sua decomposição. A patente americana US4.983.278, Pyrolysis methods with product oil recycling, de 1999. Esta patente separa as partes da borracha e gera como resultado o xisto betuminoso, a areia de alcatrão, o óleo de motor e entre outros produtos (WIPO, 2017).

\section{Adição de carga de até $60 \%$ de concentração em resina termofixa.}

Um dos mais recentes estudos protocolados junto ao INPI, sobre reciclagem de pneus é o de №. BR10 2016 005015-4, o qual narra à utilização de resíduos de pneus sendo misturados com uma concentração de resinas termofixa até a concentração de $60 \%$ (INPI, 2017).

A concentração exata dos compostos citados como matéria prima, e o método da mistura bem como a melhor rotação do misturador, o procedimento de envazamento nos moldes para cada tipo de material a ser gerado, não são discorridos na patente. Contudo, o método se mostra inovador ao usar uma concentração de pneus superior a de $30 \%$, atingindo valores até de $80 \%$. Sendo este uma inovação, já que a literatura cita que os termofixos aceitam valores em média de $30 \%$ de carga (CRQ, 2017).

O CRQ possui trabalhos publicados, que explicam sobre os polímeros e seus tipos, características técnicas, aplicações, entre outros detalhes. Mas ressalta-se que o trabalho tem um esclarecimento sobre as cargas que geralmente são minerais junto aos polímeros, entre essas cargas pode-se citar os mais comuns como: talcos, pós de mármores, quartzo, dolomita, caulim. As cargas são geralmente em pós. Destaca-se que é normal esta carga não ultrapassar a quantidade de 30\% (CRQ, 2017).

Os principais produtos gerados por este procedimento de reciclagem de pneus são exemplos os pisos, pastilhas, soleiras para janelas, entre outros itens (INPI, 2017).

\section{Método utilizado}

Para o ensaio mecânico de resistência a flexão foi utilizada a norma ASTM (2010), "Standard Test Methods for Flexural Properties of Unreinforced and Reinforced Plastics and Electrical Insulating Materials". O estudo também exigiu a utilização de um software, o qual foi utilizado o IBM SPSS STATISTICS, com a função de realizar os principais cálculos estatísticos. Estes cálculos foram efetuados pelo software se aplicando os métodos estatísticos do levantamento da normalidade, sendo estes aplicados conforme a normalidade ou a não normalidade indicado pelo estudo, e em seguida aplicou-se os métodos Sperman e Person, conforme o fato de ser ou não paramétricos e a verificação da normalidade da curva. 


\section{Ensaios mecânicos do protocolo de patente №. BR10 2016 005015-4}

Sobre o protocolo da patente $\mathrm{n}^{\circ}$. BR10 2016 005015-4, tem-se os seus ensaios mecânicos que foram feitos em laboratório independente, sendo os ensaios de resistência à flexão realizado em amostras que tinham $20 \%$ e $60 \%$ de carga de pneus em resina termofixa como matriz polimérica.

Abaixo segue descritivo do ensaio mecânico realizado, quanto à resistência do material a sua deformação ao ser submetido a esforço mecânico de flexão.

\subsection{Quanto ao ensaio de resistência à flexão}

Para estes ensaios, tem-se como primeiro passo o fato de confeccionar as amostras, a qual deve seguir padrão conforme a norma ASTM (2010) para resistência a flexão. Na figura 1 e 2, pode-se verificar o formato das amostras, as quais foram ensaiadas logo em seguida.

Figura 1: Conjunto de amostras com $20 \%$ de resíduo de borracha de pneus na matriz polimérica.

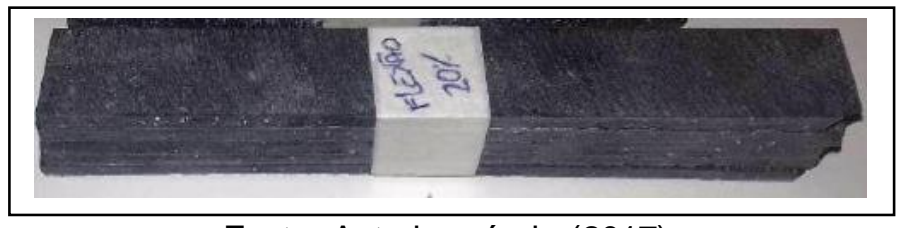

Fonte: Autoria própria (2017).

Figura 2: Conjunto de amostra com $60 \%$ de resíduo de borrachas de pneus na matriz polimérica.

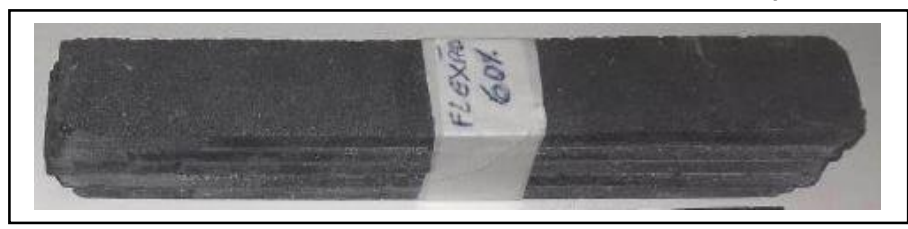

Fonte: Autoria própria (2017).

Este ensaio é usado para aquisição de dados de propriedades sob flexão para controle e especificação de materiais plásticos, assim como para caracterização qualitativa e para pesquisa e desenvolvimento. Uma das principais vantagens dos resultados deste ensaio é poder afirmar se as peças ou produtos gerados podem sofrer esforços de flexão e quanto pode ser esse esforço que os produtos vão suportar.

As propriedades sobr flexão são bastante suceptíveis à preparação da amostra e condições de ensaio, como a influência da velocidade, temperatura e umidade. $O$ ensaio seguiu a norma Standard Test Methods for Flexural Properties of Unreinforced and Reinforced Plastics and Electrical Insulating Materials (ASTM, 2010) de código D790:2010.

A temperatura usada na sala foi de $23^{\circ} \mathrm{C}$ a qual estava climatizada. A umidade estava indicando $50 \%$, e aplicação de carga de $5 \mathrm{KN}$, e se usou a velocidade do ensaio em $2 \mathrm{~mm} / \mathrm{min}$. O spam é calculado em função da espessura do corpo de prova através da seguinte equação: $L=(16 \pm 1)$.h; onde, $L$ é a distância entre dois apoios fixos (em $\mathrm{mm})$, e $\mathrm{h}$ é a espessura média dos corpos de prova utilizados $(\mathrm{mm})$.

Figura 3: Equipamento que mostra o processo de ensaio de resistência à flexão em material polimérico. 


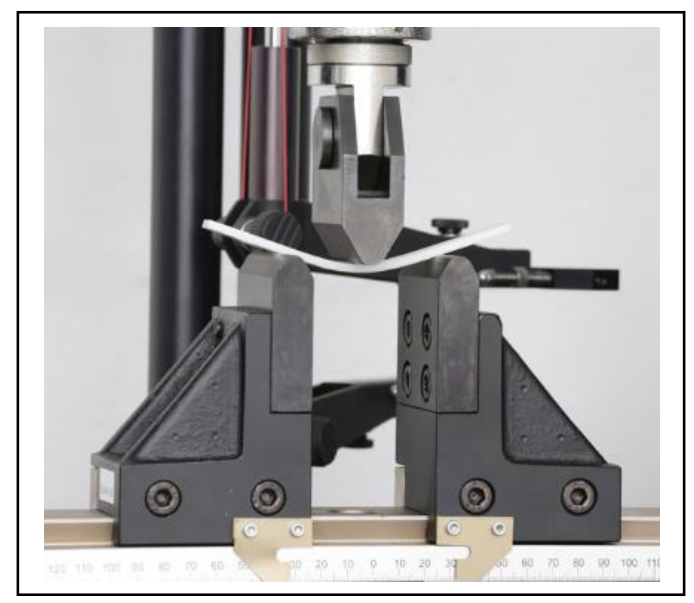

Fonte: Autoria própria (2017).

Neste ensaio de resistência a flexão, conforme a figura 3, nota-se que existe uma amostra com formato determinado, sendo um retângulo com espessura especificada conforme norma ASTM (2010). Esta amostra é colocada entre dois pontos de apoio com afastamento controlado entre eles, e localizadas mais nas proximidades da amostra. Uma punção com velocidade determinada vem sendo aplicado na região central da amostra, forçando a amostra a fazer uma flexão na sua região central, sendo a resistência do material medido a cada instante pelo equipamento. Destaca-se que são usadas 10 amostras para fazer os ensaios, e assim ter um parâmetro consistente de resultados.

\section{Resultados e discussões}

Os resultados dos ensaios de resistência à flexão estão descritos abaixo, com a aplicação dos métodos estatísticos necessários para demonstrar o objetivo já descrito anteriormente.

A primeira observação que deve ser levantada é se os dados coletados junto ao ensaio mecânico de resistência a flexão é ou não normal, e qual é a sua estatística descritiva. Observa-se os dados destacados logo abaixo conforme as tabelas 1 e 2 . Sendo que a tabela 1 registra as informações dos dados de leituras do ensaio, e a tabela 2, mostra a estatística descritiva, sua média, mediana e moda.

Tabela 1: leituras do ensaio de resistência à flexão (MPa)

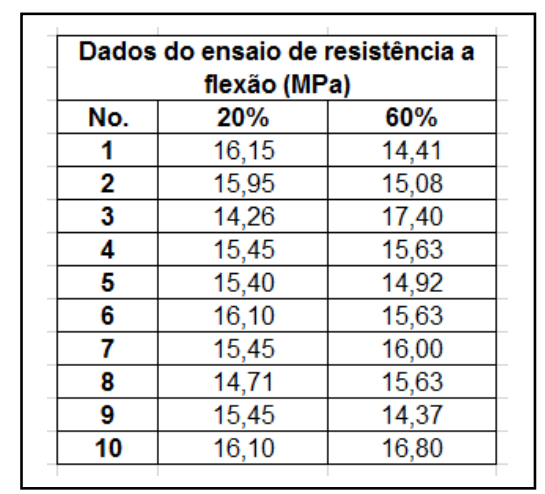

Fonte: Autoria própria (2017)

Nos dados da tabela 1, pode-se notar que a média quando a quantidade de carga de pneus na amostra é de $20 \%$, fica em $15,50 \mathrm{MPa}$, sendo a mediana de $15,45 \mathrm{MPa}$, e a 
moda é 15,45 MPa. Analisando a concentração e $60 \%$ de carga de pneus usados, tem-se que a média é de 15,61 MPa, e a mediana tem seu valor de 15,63 MPa, enquanto a moda é de 15,63 MPa. Na tabela 2, exemplifica os dados para melhor compreensão e avaliação.

Tabela 2: Estatística descritiva referente aos dados do ensaio de resistência a flexão (MPa)

\begin{tabular}{|ll|r|r|}
\hline & & \\
\hline$N$ & Valid & 10 & 10 \\
& Missing & 1 & 1 \\
Mean & 15,5020 & 15,6170 \\
Median & 15,4500 & 15,6300 \\
Mode & 15,45 & 15,63 \\
\hline
\end{tabular}

Fonte: Autoria própria (2017)

O sumário dos dados obtidos pelo ensaio de resistência a flexão se faz necessário para validação dos dados e posicionamento do estudo. Na tabela 3, há o descritivo dos casos validos, do percentual analisado pelo programa IBM SPSS STATISTICS 23.

Tabela 3: Sumário do processamento dos dados referente ao ensaio de resistência à flexão

\begin{tabular}{|c|c|c|c|c|c|c|}
\hline & \multicolumn{6}{|c|}{ Cases } \\
\hline & \multicolumn{2}{|c|}{ Valid } & \multicolumn{2}{|c|}{ Missing } & \multicolumn{2}{|c|}{ Total } \\
\hline & $\mathrm{N}$ & Percent & $\mathrm{N}$ & Percent & $\bar{N}$ & Percent \\
\hline ResFlex20 & 10 & $100,0 \%$ & 0 & $0,0 \%$ & 10 & $100,0 \%$ \\
\hline ResFlex60 & 10 & $100,0 \%$ & 0 & $0,0 \%$ & 10 & $100,0 \%$ \\
\hline
\end{tabular}

Fonte: Autoria própria (2017)

Com os mesmos dados do ensaio de resistência a flexão, e fazendo uma aplicação via histograma, tem-se um perfil estatístico sobre a normalidade com cada porcentual de adição de resíduos de pneus na matriz polimérica.

A tabela 4 registra o perfil desta normalidade, através de algumas informações relevantes como a média já mostrada anteriormente na tabela 2, a qual é 15,50 para 20\% de carga e $15,61 \%$ para $60 \%$ de carga de pneus junto a matriz polimérica. 
Tabela 4: Medidas de tendência central do ensaio de resistência à flexão

\begin{tabular}{|c|c|c|c|c|}
\hline & & & Statistic & Std. Error \\
\hline \multirow[t]{13}{*}{ ResFlex20 } & Mean & & 15,5020 & 19788 \\
\hline & \multirow{2}{*}{$\begin{array}{l}95 \% \text { Confidence Interval } \\
\text { for Mean }\end{array}$} & Lower Bound & 15,0544 & \\
\hline & & Upper Bound & 15,9496 & \\
\hline & \multicolumn{2}{|l|}{$5 \%$ Trimmed Mean } & 15,5350 & \\
\hline & \multicolumn{2}{|l|}{ Median } & 15,4500 & \\
\hline & \multicolumn{2}{|l|}{ Variance } &, 392 & \\
\hline & \multicolumn{2}{|l|}{ Std. Deviation } &, 62576 & \\
\hline & \multicolumn{2}{|l|}{ Minimum } & 14,26 & \\
\hline & \multicolumn{2}{|l|}{ Maximum } & 16,15 & \\
\hline & \multicolumn{2}{|l|}{ Range } & 1,89 & \\
\hline & \multicolumn{2}{|l|}{ Interquartile Range } & 87 & \\
\hline & \multicolumn{2}{|l|}{ Skewness } &,- 921 & ,687 \\
\hline & \multicolumn{2}{|l|}{ Kurtosis } &, 280 & 1,334 \\
\hline \multirow[t]{13}{*}{ ResFlex60 } & \multicolumn{2}{|l|}{ Mean } & 15,6170 & ,30929 \\
\hline & \multirow{2}{*}{$\begin{array}{l}95 \% \text { Confidence Interval } \\
\text { for Mean }\end{array}$} & Lower Bound & 14,9173 & \\
\hline & & Upper Bound & 16,3167 & \\
\hline & \multicolumn{2}{|l|}{$5 \%$ Trimmed Mean } & 15,5872 & \\
\hline & \multicolumn{2}{|l|}{ Median } & 15,6300 & \\
\hline & \multicolumn{2}{|l|}{ Variance } & 957 & \\
\hline & \multicolumn{2}{|l|}{ Std. Deviation } & ,97807 & \\
\hline & \multicolumn{2}{|l|}{ Minimum } & 14,37 & \\
\hline & \multicolumn{2}{|l|}{ Maximum } & 17,40 & \\
\hline & \multicolumn{2}{|l|}{ Range } & 3,03 & \\
\hline & \multicolumn{2}{|l|}{ Interquartile Range } & 1,41 & \\
\hline & \multicolumn{2}{|l|}{ Skewness } & .478 & ,687 \\
\hline & \multicolumn{2}{|l|}{ Kurtosis } &,- 295 & 1,334 \\
\hline
\end{tabular}

Fonte: Autoria própria (2017)

Contudo, as informações que mais ilustram a tabela 4 são quanto à assimetria e a curtose. Os dados quanto à assimetria têm resultados para $20 \%$ e $60 \%$ respectivamente iguais a $-0,921$ e 0,478 . Os resultados quanto a curtose com $20 \%$ de carga de pneus reciclado é de 0,280, e com $60 \%$ de carga de pneu, a curtose é igual a -0,295.

Tabela 5: Teste de normalidade

\begin{tabular}{l}
\begin{tabular}{|l|r|r|r|r|r|r|}
\hline & \multicolumn{3}{|c|}{ Kolmogorov-Smirnov $^{\text {a }}$} & \multicolumn{3}{|c|}{ Shapiro-Wilk } \\
\cline { 2 - 7 } & Statistic & \multicolumn{1}{c|}{ df } & \multicolumn{1}{c|}{ Sig. } & Statistic & \multicolumn{1}{c|}{ df } & \multicolumn{1}{c|}{ Sig. } \\
\hline ResFlex20 &, 235 & 10 &, 124 &, 872 & 10 &, 106 \\
ResFlex60 &, 148 & 10 &, $200^{*}$ &, 950 & 10 &, 673 \\
\hline
\end{tabular} \\
*. This is a lower bound of the true significance. \\
a. Lilliefors Significance Correction \\
\hline
\end{tabular}

Fonte: Autoria própria (2017)

Na tabela 5 interpreta-se que os dados são menores do que 30 amostras, portanto considerados poucos, sendo 10 amostras em cada categoria da análise, e isto justifica a aplicação da metodologia de Shapiro-Wilk, descartando os dados de KolmorogovSmirnov.

Observa-se que a significância (sig.) de 0,106 para $20 \%$ de resistência a flexão (ResFlex20), e a significância (sig.) de 0,673 para $60 \%$ de resistência a flexão (ResFlex60). 
Ao se observar os dados quando posicionados na forma do histograma, há percepção dos resultados das leituras dos ensaios realizados e suas distorções, bem como similaridade.

Figura 4: Frequência dos resultados sendo: a) $20 \%$ e b) $60 \%$ de carga de pneus na matriz polimérica.

a)

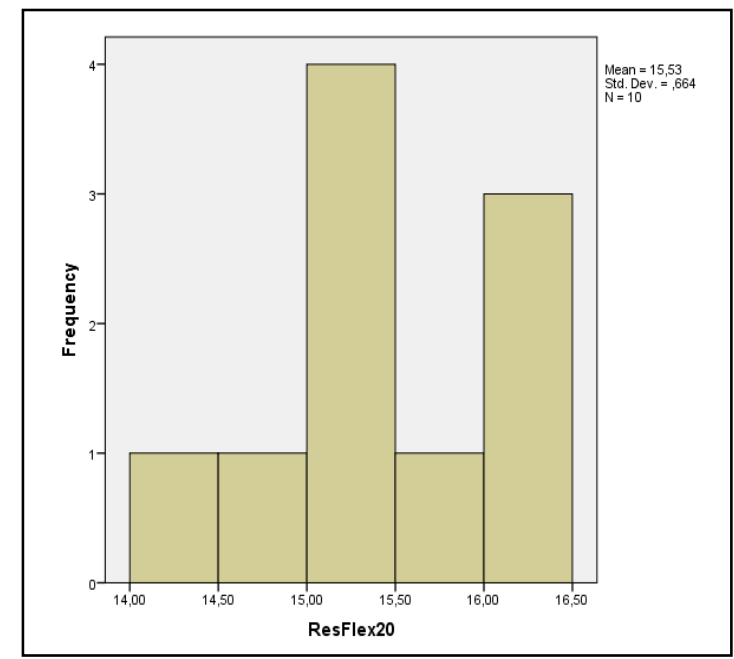

b)

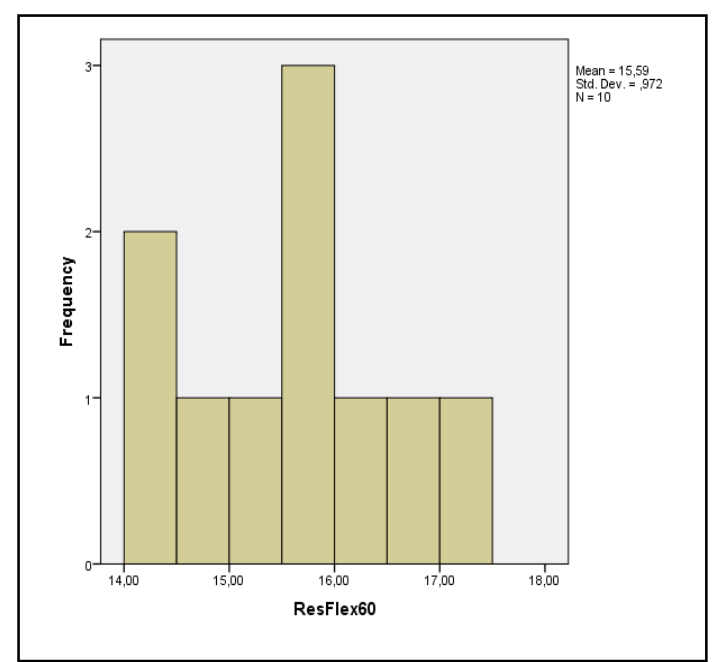

Fonte: Autoria própria (2017)

Uma significativa comparação dos resultados é feita ao observar os dados quando se tenta contrastar os valores ao se ter as retas formadas com os valores de leituras conforme as porcentagens dos resíduos de pneus formando as amostras, conforme a figura 5 , abaixo.

Figura 5: Resultado do ensaio de resistência à flexão com a) $20 \%$ e b) $60 \%$ de carga de pneu respectivamente

a)

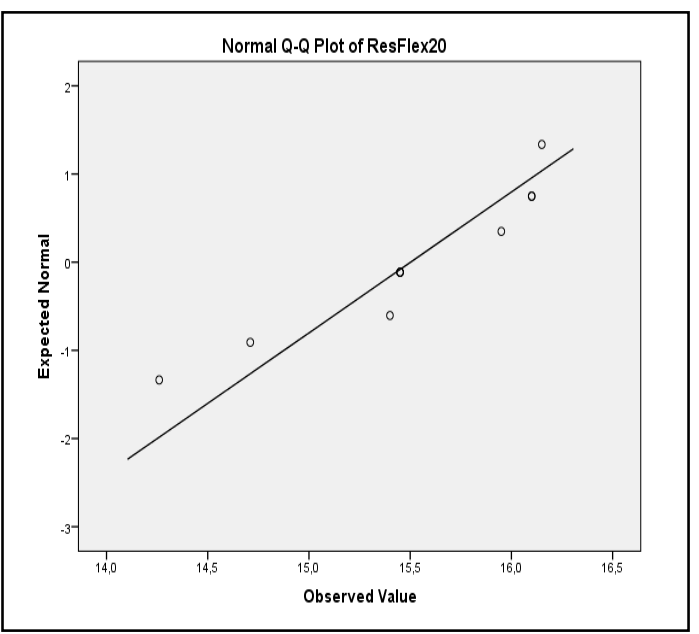

b)

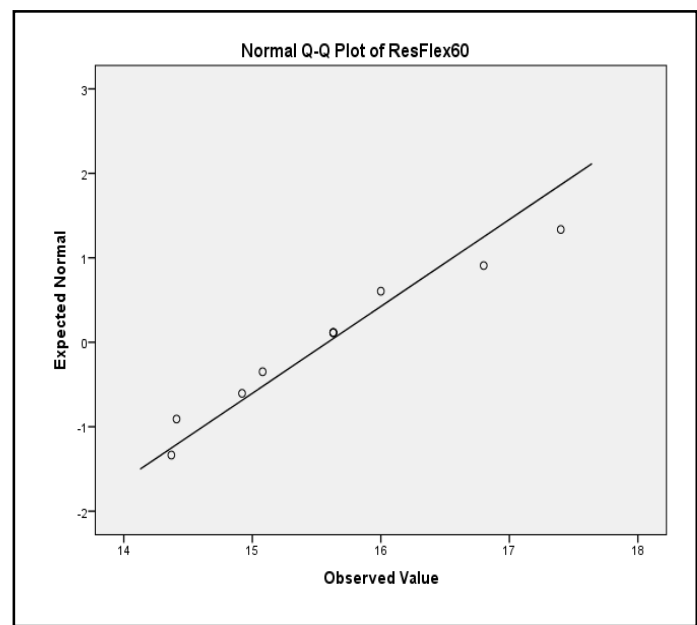

Fonte: Autoria própria (2017)

Com os dados estruturados é possível realizar uma comparação entre as variáveis envolvidas, possibilitando uma visão se há linearidade, se tem aspecto positivo ou tendência negativa.

A figura 6 mostra este perfil dos dados, favorecendo uma possível comparação entre as variáveis, e análise da correlação dos dados pesquisados. 
Figura 6: Gráfico para correlação dos dados

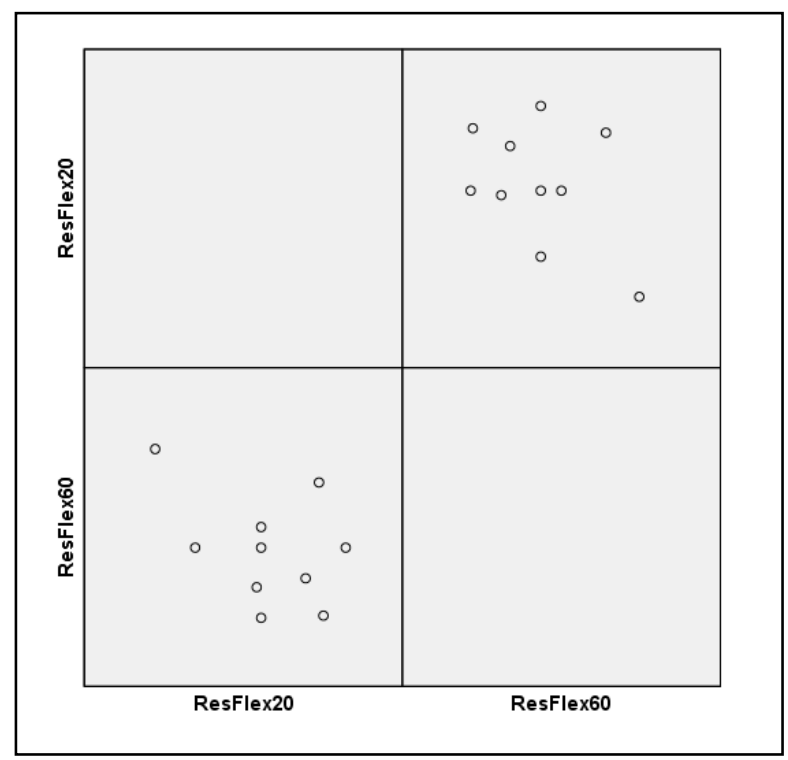

Fonte: Autoria própria (2017)

Um dos principais resultados das leituras efetivadas junto ao ensaio de resistência mecânica é a correlação entre os dados. Na tabela 6, se têm as informações paramétricas, descritas pelo método de Pearson, que segue a distribuição normal e simétrica, onde contém menos de 30 amostras.

Tabela 6: Correlação de Pearson para 20\% e 60\% de carga de pneus inservíveis

\begin{tabular}{|ll|r|r|}
\hline & & ResFlex20 & ResFlex60 \\
\hline ResFlex20 & Pearson Correlation & 1 &,- 406 \\
& Sig. (2-tailed) & &, 244 \\
& N & 10 & 10 \\
\hline ResFlex60 & Pearson Correlation &,- 406 & 1 \\
& Sig. (2-tailed) &, 244 & 10 \\
& N & 10 & 10 \\
\hline
\end{tabular}

Fonte: Autoria própria (2017)

O método de Spearman é para caso não paramétrico e de curva não normal. Abaixo segue análise de correlação para analisar diante uma comparação com os dados de significância encontrados e para efetuar uma comparação com os dados do método de Pearson.

Tabela 7: Correlação pelo método de Spearman

\begin{tabular}{|lll|r|r|}
\hline & & & ResFlex20 & ResFlex60 \\
\hline Spearman's rho & ResFlex20 & Correlation Coefficient & 1,000 &,- 198 \\
& & Sig. (2-tailed) & $\cdot$ &, 584 \\
& $\mathrm{~N}$ & 10 & 10 \\
\cline { 2 - 5 } & ResFlex60 & Correlation Coefficient &,- 198 & 1,000 \\
& Sig. (2-tailed) &, 584 &. \\
& $\mathrm{~N}$ & 10 & 10 \\
\hline
\end{tabular}

Fonte: Autoria própria (2017) 
Há conforme o método de Spearman um coeficiente de correlação entre ResFlex20 e ResFlex60, de -0,198 com significância de 0,584.

\section{Conclusões}

Aplicando a estatística descritiva, pode-se ter resultados como os dos ensaios de resistencia a flexão. $O$ melhor resultado deste ensaio mecânico foi com o material com $60 \%$ de resíduo de pneu adicionado na matriz polimérica; o qual suportou com a carga média de 15,61 MPa. Este resultado se deve a grande presença da porcentagem de particular de pneus diluído na matriz polimérica, vindo a absorve a carga aplicada na matriz.

A média mostrou poder ser aplicada uma vez que não existe uma distorção significativa entre os valores da média com a mediana e a moda, podendo ser observado na tabela 2, tais resultados indiferente da concentração da quantidade de pneus disolvido na matriz das amostras, convalidando assim o valor da média como sendo a principal referência do estudo.

Ao aplicar a estatística para se avaliar a normalidade dos resultados, constata-se que os resultados são todos normais, pois apresentam um valor de $\mathrm{P}>0,05$, conforme se pode notar na tabela 5, justificando a aplicação do método de Shapiro-Wilk e de Pearson. Também se pode concluir, neste caso, que as informações caracterizam serem paramétricas.

Os gráficos de histogramas visto na figura 4 confirmam a distribuição dos resultados como baixa distorção, e conclui-se pela sua similaridade.

Sobre a figura 5 , pode-se concluir que é linearidade dos dados e similaridade entre as retas formadas tanto com $20 \%$ e com $60 \%$ de carga adicional de pborracha de pneu na matriz polimérica. Outro aspecto é que a reta demonstra ter correlação dos dados serem negativos e com aspecto positivo.

$\mathrm{Na}$ figura 6 , conclui-se que há baixa dispersão dos dados medidos sobre a resistência a flexão, sendo paramétrica, confirmando que há correlaçào entre os ados coletados, que segue a distribuição normal e por isto se indica a leitura por Pearson, onde estes dados são simétricos.

A tabela 6 relata dados que geram a conclusão de que a resistência à flexão com $20 \%$ de carga tem correlação com a resistência à flexão com $60 \%$ de carga de pneu, tendo uma significância positiva $(0,673)$, com correlação a tendência negativa e de força forte.

O teste de Spearman é não paramétrico e o teste realizado com KolmogorovSmirnov é indicado amostras superiores a 30 unidades. E pelos resultados obtidos podese concluir que os dois métodos não são indicados para a leitura dos dados realizados nas amostras tanto com $20 \%$ como com $60 \%$ de carga para a resistência a flexão.

A conclusão é que todas as leituras efetuadas e com as percepções das análises, o material tem suas leituras dos ensaios correlatos e com perfil condizente um com o outro, indiferente da concentração da quantidade de resíduos de pneus adicionado na mistura química; levando a interpretar de que os valores sugerem aplicações práticas, pois a resistência à flexão é validada pelos dados e métodos estatísticos aplicados.

A maior conclusão obtida é o fato de que o melhor resultado de resistência a flexão foi atingido com as amostras com $60 \%$ de carga de resíduos de pneus, o que gera a afirmação de que se trata de algo inédito e inovador, já que a literatura (CRQ, 2017), cita que a carga máxima permitida está em torno de 30\%, e neste caso pode-se determinar que quanto maior a carga de borracha de pneus junto a matriz polimérica de um termofixo utilizando a técnica da patente BR10 2016 005015-4 tem-se resultados superiores quanto à resistência à compressão do que quando a matriz possui pouca 
adição de carga. Esta conclusão favorece a determinação de que placas confeccionadas com carga de $60 \%$ de resíduos de pneus podem ser utilizadas para fabricar produtos que suportem bem a resistência à compressão conforme ensaio indicado. Com isto o objetivo geral foi respondido ao se obter tais conclusões citadas e aqui destacadas.

\section{Referências}

ASTM - American Society for testing and materials. Standard Test Methods for Flexural Properties of Unreinforced and Reinforced Plastics and Electrical Insulating Materials. Norma D790, 2010. Disponível em: <https://www.astm.org/DATABASE.CART/HISTORICAL/D790-10.htm>. Acesso em: 15 dez. 2017.

BERTOLLO, S. M.; FERNANDES, J. L. J.; SCHALCH, V. Benefícios da incorporação de borracha de pneus em pavimentos asfálticos. In: CONGRESSO INTERNACIONAL DE ENGENHARIA SANITÁRIA E AMBIENTAL, 28., 2002, Cancun. Anais... Cancun: AIDIS, 2002.

CRQ - Conselhor Regional de Química. Polímeros termoplásticos, termofixos e elastômeros. Minicurso CRQ, São Paulo, Ago. 2017. Disponível em:<http://www.crq4.org.br/sms/files/file/apostila_pol\%C3\%ADmeros_0910082013_site.p df. >. Acesso em: 03 nov. 2017.

BRITO, M. P. Managing Reverse Logistics or Reversing Logistics Management? 2004. $327 \mathrm{f}$. Tese (Doutorado em Research in Management) - Programa de Pós g Graduação em Research in Management, Erasmus University Roterdam, Holanda.

EPA - Environmental Protection Agency. State Scrap Tire Programs - A Quick Reference $\quad$ Guide. $1999 . \quad$ Disponível em: < http://www.asphaltrubber.org/ARTIC/Reports/RPA A1006.pdf>. Acesso em: 20 fev. 2019.

JON, A. Uses of Recycled Rubber Tires in Highways. Washington: TRB - National Research Council, 1994.

FHWA - Federal Highway Administration. Crumb Rubber Modifier: Design Procedures and Construction Practices. Workshop Notes, Department of Transportation, 1993. Disponível em: <https://www.fhwa.dot.gov/pavement/pubs/013377.pdf>. Acesso em: 01 mar. 2018.

FREIRE, F. G. M.; GUEDES, A. P. S. A gestão do sistema logístico inverso para pneusresíduo e sua relação com a eficácia e eficiência. In: ENCONTRO NACIONAL DE ENGENHARIA DE PRODUÇÃO, 26., 2006, Fortaleza. Anais... Fortaleza: ABEPRO, 2006.

INPI - Instituto Nacional de Propriedade Intelectual. Patente. 2017. Disponível em: $<$ https://www.inpi.com.br>. Acesso em: 13 jan. 2017.

LAGARINHOS, C. A. F.; TENÓRIO, J. A. S. Reciclagem de pneus: discussão do impacto da política brasileira. Engevista, Rio de Janeiro, v. 11, n. 1, p. 32-49, jul. 2009. 
LAGARINHOS, C. A. F.; TENÓRIO, J. A. S. Logística reversa dos pneus usados no Brasil. Polímeros, São Carlos, v. 23, n. 1, p. 49-58, oct. 2013.

LENS. Banco Mundial de Patentes Abertas da Cambia e da Universidade de Tecnologia de Queensland. 2017. Disponível em: <https://www.lens.org/lens>. Acesso em: 20 dez. 2017.

RAMIREZ, C.; SCHAU, C.; EMMIOĞLU, E. The importance of attitudes in statistics education. Statistics Education Research Journal, Voorburg, v. 11, n.2, p. 57-7, nov. 2012.

RECHHOLD DO BRASIL S/A - Fabricante de resina no Brasil. Disponível em http://www.rechhold.com. Acesso em 18 de julho de 2018.

RODRIGUES, M. R. P. Caracterização e utilização do resíduo da borracha de pneus inservíveis em compósitos aplicáveis na construção civil. 2008. 290 f. Tese (Doutorado em Ciências da Engenharia Ambiental) - Programa de Pós-Graduação em Ciências da Engenharia Ambiental, Universidade de São Paulo, São Carlos.

SARACEVIC, T. Relevance: a review of the literature and a framework for thinking on the notion in information science. Part III: behavior and effects of relevance. Journal of the Association for Information Science, North Carolina, v. 58, n. 13, p. 1915-1933, oct. 2007

UNDRI, A. et al. Microwave pyrolysis of polymeric materials: waste tires treatment and characterization of the value-added products. Journal of Analytical and Applied Purolysis, v. 103, n. 1, p. 149-158, sep. 2013.

WIPO. Organização Mundial da Propriedade Intelectual. Patente. 2017. Disponível em <https://www.wipo.int>. Acesso em 20 dez. 2017. 